\title{
Función de la comunicación interpersonal en la calidad de servicios empresariales
}

\author{
Susana GONZALES ACOSTA
}

La presión competitiva ha obligado a muchas organizaciones a redescubrir al consumidor y a evaluar su capacidad de satisfacer las necesidades. Además, la creciente presión de los consumidores y de la competencia están obligando a que las organizaciones, en especial las de servicio, presten una detenida atención al tema de la calidad.

Las organizaciones de servicios reconocen la necesidad de algún cambio, pero aún hay lentitud en la comprensión de la naturaleza de ese cambio. Se cree que con sólo brindar una atención esmerada en los niveles más bajos de la organización se logran cambios fundamentales en ésta y se está preparado para competir en el mercado. Es obvio que hay que tener en cuenta otros factores para alcanzar el nivel de calidad que se requiere; uno de ellos es el de la comunicación.

La comunicación sirve como vehículo indispensable para ampliar la clientela, conseguir su lealtad, motivar a los empleados y darles una idea exacta de las normas de calidad que deben respetar. La comunicación es el único medio de hacer conocer, a priori, la diferencia y la ventaja de la empresa en relación con sus competidores.

Los aspectos que analizaremos a lo largo de este ensayo permitirán entender la forma en que la comunicación interpersonal interviene como condición necesaria para el desarrollo de la calidad de servicios en una organización. 


\section{LA ORGANIZACIÓN COMO SISTEMA Y SU RELACIÓN CON LA CALIDAD DE LOS SERVICIOS}

Las organizaciones existen y se desarrollan en un contexto, en un ambiente, que se encuentra condicionado por el suprasistema (político, económico, social y cultural) de cada país o región.

Actualmente nuestras organizaciones viven inmersas en la globalización del mercado, en el rápido desarrollo tecnológico, con una competencia cada vez mayor y enfrentándose cada vez más a clientes más exigentes que cuentan con mayor información.

Sobrevivir en este entorno no es tarea fácil. No basta tener los recursos materiales necesarios, los financieros apropiados, ni los tecnológicos o humanos. La empresa debe además ser capaz de adaptarse a un cambio constante y rápido, y aprovechar al máximo la información que le suministra el entorno.

Analizar las organizaciones teniendo en cuenta la Teoría General de Sistemas implica:

1. Tolela Myers, Michelle. Administración mediante la comunicación. Un enfoque organizacional, México, 1983, p. 47.

2. Kullmer, Jorge. Gerencia No 201, marzo-abril de 1993, p. 29.
“... ver el énfasis en la estructura, la interdependencia y las relaciones, sólo se ocupa de las partes de un sistema en la medida en que se relacionan con el todo, esta perspectiva considera a la comunicación como un proceso esencial que permite la interdependencia entre las partes de cualquier sistema" 1 .

No debemos olvidar que lograr competitividad en el mercado actual requiere plantear como objetivo principal alcanzar la calidad entendida como:

\footnotetext{
"la estrategia que permite a las empresas disponer del conocimiento de todos los empleados de una manera sistemática, de mejorar la eficiencia de los procesos, mediante el uso de técnicas (...) y de tener clientes satisfechos a través de una continua búsqueda de sus necesidades y requerimientos" 2 .
}

La "calidad es asunto de todos" y comprende a todas las partes que forman el sistema. Tal afirmación es aplicable en una organización de servicios, en la que se debe estar consciente de esta necesidad, ya que si uno de los subsistemas no funciona, el sistema se verá seriamente afectado, debido a la interdependencia que significa la influencia mutua y reciproca entre las partes del todo. 
Los clientes, entendidos como los individuos que compran bienes o servicios, forman parte del sistema y son una valiosa fuente de información, insumo básico de un sistema, pues a través de sus gustos o necesidades proporcionan señales sobre su ambiente y sobre su propio funcionamiento en ese ambiente, y esto se logrará a través de la comunicación.

\section{LA COMUNICACIÓN Y EL PROCESO DE SERVICIOS}

Lo antes expuesto nos hace deducir que un sistema consta de partes interrelacionadas; lo que le sucede a una parte indefectiblemente afecta a todas las demás. Lo importante es la relación que cada uno de esos elementos tiene con los demás y cómo se influyen mutuamente.

A través de un ejemplo analizaremos lo indicado: Un cliente espera ser atendido por el cajero terminalista de un banco y lleva 20 minutos en una fila. Al momento de ser atendido se le señala que tiene que esperar un momento porque su cheque pasa a consulta; luego de 20 minutos de espera se le comunica que lamentablemente no se puede cambiar su cheque porque el sectorista, persona que tiene que aprobar esa transacción, no se encuentra y no saben cuánto se demorará. El cliente, evidentemente fastidiado exige que se le resuelva el problema. Luego de una serie de quejas se informa al administrador de la agencia, quien luego de 10 minutos más de espera soluciona el problema.

Mediante este ejemplo queremos demostrar que el proceso de comunicación es una transacción donde los emisores pasan a ser receptores y viceversa, logrando la interdependencia entre todos los elementos interactuantes como producto de los comportamientos comunicativos previos en el sistema. Sin embargo, debemos señalar que lo más importante es la manera como éste se considera para dar forma a los comportamientos comunicativos.

Si bien es cierto que la organización que presta servicios es responsable de su calidad, también es cierto que más responsabilidad tiene el individuo que presta el servicio, por lo tanto, el cliente no tiene otra alternativa que la de considerar al empleado como el responsable de la calidad de atención recibida "en cualquier contacto, encuentro o transacción el cliente es muestra de uno (...) Un cliente tiene que pasar por contactar con dos o tres personas antes de contactar con la persona adecuada. La calidad viene determinada por la conducta y actitudes de las muestras individuales"3.
3. Rossander, A.C. La búsqueda de la calidad en los servicios. Ediciones Díaz de Santos, 1992, p. 20. 
4. Luft, Joseph. La interacción humana. National Press Book, 1969.
Se genera un conjunto de expectativas en el cliente que va en busca de satisfacción de una necesidad. Hay que evaluar el servicio que espera el cliente, el servicio que recibe y el que percibe.

Cada persona percibe la realidad de manera diferente y cada uno evalúa su comportamiento y el de los demás, basándose en sus percepciones, y lo más importante es que cada cual actúa de acuerdo con la forma como ve las cosas; este es el motivo por el cual la gente cambia cada vez más de bancos, por ejemplo, porque está cansada de esperar en la ventanilla.

Apreciar esta interacción comunicativa es más fácil si se aplica el modelo denominado "Las Ventanas de Johari" propuesto por Joseph Luft, en su libro La interacción humana ${ }^{4}$. El autor plantea que en toda acción comunicativa entre dos personas se da un intercambio tanto a nivel intrapersonal como a nivel interpersonal.

\begin{tabular}{l|l|l}
\hline & Conocido para uno & Desconocido para otro \\
\hline $\begin{array}{l}\text { Conocido } \\
\text { para otros }\end{array}$ & 1. Espontáneo & 2. Ciego \\
\hline $\begin{array}{l}\text { Desconocido } \\
\text { para otros }\end{array}$ & 3. Oculta & 4. Desconocido \\
\hline
\end{tabular}

Como se observa en el cuadro, la ventana consta de cuatro cuadrantes.

Cada cuadrante representa a la persona en su totalidad cuando entra en relación con otra(s) persona(s). La línea que divide los cuadrantes se refiere a las motivaciones, los sentimientos y lo consciente que pueda o no estar uno respecto de su conducta.

Cada uno de los cuadrantes debe ser entendido, además, de la siguiente forma:

\section{Cuadrante espontáneo}

Está referido a la congruencia que existe entre la experiencia, la conciencia y la comunicación. Es decir, cuando los sentimientos, motivaciones y conductas son conocidos por unos y otros. 


\section{Cuadrante ciego}

Lo apreciamos cuando se da una falta de congruencia entre la conciencia y la experiencia. Ello ocurre cuando la conducta, los sentimientos y las motivaciones son conocidos para los otros, pero desconocidos para la persona.

En este cuadrante se transmite contenidos afectivos a través de la comunicación no verbal. Lamentablemente este es el tipo de comunicación que se utiliza con mayor frecuencia "de ahí que los problemas de comunicación no nacen de que comuniquemos inadecuadamente, sino de que comunicamos demasiado adecuadamente" 5 . Puesto que además de los significados que se les da a las palabras, los movimientos del cuerpo juegan una parte tan importante como las palabras. El significado de lo que se dice en palabras o por escrito es sólo una parte, a veces pequeña, de lo que se quiere comunicar o expresar.

\section{Cuadrante oculto}

En éste falta congruencia entre la conciencia y la comunicación. La persona oculta algo a su interlocutor, lo que se puede dar por diversas razones, desde por respeto a los demás hasta por hipocresía. Este cuadrante, al igual que el anterior, también se utiliza con frecuencia,

\section{Cuadrante desconocido}

Este es un cuadrante de incongruencia total; es desconocido tanto por la persona como por los otros. Es accesible sólo a través de procedimientos sicológicos especiales.

$\mathrm{Si}$ todo esto lo relacionamos con nuestro punto de interés podemos afirmar que la calidad de servicio está condicionada por las actitudes y comportamientos de una sola persona o grupo de personas que tienen contacto con el cliente. Por ejemplo si nos ubicamos en el cuadrante dos de la ventana, nos daremos cuenta de la falta de congruencia que se da entre la experiencia y la conciencia. Igualmente, el cliente se dará cuenta de esta incongruencia a través de los gestos y del tono de voz que se manifiesta cuando se le presta el servicio.

Por lo general, señala Rossander, "son los empleados peor pagados en la compañía los que prestan servicio"6. No se justifica una mala calidad de servicio porque la tarea la realizan empleados mal pagados y muy buena porque la rea-

6. Rossander, A.C. Op. cit., p. 48. 
7. Terry y Franklin. Principios de Administración, México: Compañía Editorial Continental, 1986. lizan empleados bien pagados, sino que, al contrario, implica que existe una mayor preocupación por el aprendizaje en los procedimientos del trabajo y en las actitudes esperadas hacia los clientes.

La percepción en esta relación juega un papel importante; la manera en que la persona piensa influye sobre lo que percibe y viceversa. Como la calidad no puede determinarse de antemano el cliente no sabe si el servicio será o no será satisfactorio.

Es en ese momento cuando el cliente realiza una selección de acuerdo con la interacción social, para luego hacer la evaluación correspondiente; estas experiencias serán almacenadas por la persona. El individuo, después, tendrá acceso a sus experiencias recordándolas cada vez que lo desee.

La comunicación no verbal, como la que se realiza con los músculos faciales cuando se sonrie o cuando se frunce el ceño, los ademanes y las diferentes posturas del cuerpo muestran una gama de sentimientos.

También cumple un papel la presencia de lo icónico en este proceso de comunicación, como las diferentes señales que se aprecian en un local de comida rápida; por ejemplo, los uniformes, la disposición de las sillas, la decoración, el material que se utiliza para atender al cliente, etc.

Todos estos comportamientos comunicativos se emplean simultáneamente y cada uno califica de alguna manera el significado del otro.

Otro aspecto importante que interviene en este proceso es la motivación. Según Terry y Franklin "la motivación es la necesidad o impulso interno del individuo que lo mueve hacía una acción orientada a un objetivo"7. Algunas de las teorias más utilizadas en este campo son la Teoría de la Jerarquía de Necesidades de A. Maslow y la Teoría de los Dos Factores de F. Herzberg.

Para Maslow hay una tendencia natural al crecimiento y desarrollo personal que nos permite capacitar al individuo para que perciba necesidades (jerarquizadas) y se motive para su logro en sentido ascendente; de acuerdo con las oportunidades que brinde su entorno, para Herzberg, una persona necesita tener un trabajo cuyo contenido sea desafiante, estar en verdad motivado, aunque la ausencia de motivadores no necesariamente trae consigo insatisfacción.

El hecho de que a un empleado se le brinde las condiciones ambientales fisicas necesarias para que realice una labor no garantiza que éste se encuentre motivado. Sin embargo, en muchas empresas se comete el error de suponer que si el empleado reduce su nivel de producción o 
realiza una labor inadecuada es porque no está lo suficientemente motivado y la única manera de incrementar su nivel de motivación es arreglando la oficina o aumentándole de sueldo, lo que Herzberg denomina "factores higiénicos", que evitan la insatisfacción más no motivan. Se olvidan muchas veces que el empleado tiene deseos de superación, que necesita retos o una palabra de aliento, lo que Herzberg denomina "factores motivadores" y Maslow lo describe en sus últimos niveles de necesidades (autorrealización).

La motivación tiene una participación activa en el proceso de comunicación interpersonal. En la medida en que se logra satisfacción con la labor que se realiza se podrá canalizar el talento y la energía hacia los objetivos de la organización, logrando una comunicación más eficaz.

La comunicación interpersonal en el servicio no sólo está condicionada por los factores antes mencionados, sino que además intervienen factores ambientales, culturales, educativos y sicológicos, de los cuales no se ha realizado un detenido análisis teórico.

\section{LA RETROALIMENTACIÓN Y EL CLIENTE}

La retroalimentación se puede definir como la información que nutre a la organización proporcionándole señales sobre su ambiente y funcionamiento. "El tipo de respuesta, las formas de interacción y la evaluación de las interacciones son puntos críticos para usarse retroalimentando y generando el cambio, partiendo de la comunicación proveniente del lado externo..." 8 .

En general, la retroalimentación permitirá determinar si el mensaje ha sido enviado y recibido como se pretendía.

Según nuestro punto de interés, los clientes miden la actuación de una organización mediante el servicio. Esto significa que la retroalimentación se realiza casi de forma inmediata; el brindar servicio implica tener cuidado en detectar las necesidades de nuestros clientes y tomar las acciones correspondientes para satisfacerlas de manera óptima. Esto implica cambiar las formas de pensar de cada uno de los componentes de la organización; por ejemplo, podemos empezar pensando que cada empleado es cliente del trabajo que realiza su compañero y que tiene todo el derecho de exigir un buen trabajo y un buen trato y a su vez tiene la obligación de responder con la misma actitud; esto se verá reflejado en la atención que se brinde al cliente que se acerca a la organización.
8. Tello, Max et al. "Empresa y comunicación. Estado de la comunicación empresarial en el Perú", en Cuadernos Cicosul No ${ }^{\circ}$ 17. Lima: Universidad de Lima, 1994, p. 42. 
La educación y la capacitación que se ofrezca en los diferentes niveles de la organización, así como las normas y procedimientos correctamente establecidos, contribuirán a alcanzar el objetivo de lograr calidad.

La retroalimentación nos permite incluso reafirmar o modificar la imagen corporativa de la empresa, ya que consciente o inconscientemente toda empresa tiene una identidad o proyecta algún tipo de imagen. Para explicar este punto realizaremos una comparación con las personas; por lo general otorgamos atributos positivos a aquellas cosas que nos son familiares y a la inversa a las cosas menos conocidas, de ahí que la reputación juega un papel importante. Una buena reputación como parte de la imagen corporativa tendrá un efecto mayor sobre la decisión de las personas.

La imagen corporativa y la reputación que tenga una organización está en relación con su cultura organizacional, entendida como un patrón de conducta, de valores, de normas, de pensamiento y de acción, que está presente en una sociedad, y que se hereda transmitiéndose de generación en generación. La cultura se aprende, permanece y perdura en el tiempo.

Dos autores nos ilustran desde el punto de vista económico y político-social acerca de las culturas en los negocios. Deal y Kennedy en su libro Culturas corporativas distinguen cuatro tipos de cultura organizacional formada por distintas formas de competencia que se dan en los ambientes empresariales.

En ambientes de negocio donde los riesgos que se corren son altos y constantes, pero donde hay rápida retroalimentación y conocimiento sobre el impacto de las decisiones en el mercado y sobre la empresa, se desarrolla una cultura fuerte, dura, de lucha, flexible y que responde a la economía y al mercado. Aquí hay relaciones efimeras, trato amable pero no se establecen fuertes lazos. Es una cultura de tipo machista (construcción, cosmética, publicidad).

En ambientes en que los riesgos que se corren son bajos, pero donde hay rápida retroalimentación sobre los resultados, la cultura será de fuerte competitividad, de acciones rápidas, pero no destructivas, con gran orientación al cliente y relaciones internas de amistad y juego (de pasarlo bien). Es una cultura de tipo trabajo duro pero entretenido (comercio, menudeo, consumo masivo, computación).

En ambientes de altísimo riesgo, que involucran grandes inversiones, pero donde toma mucho tiempo conocer los resultados de las acciones de la empresa, se genera una cultura de trabajo perseverante, de no mucha agitación, donde 
todo se enfoca hacia el futuro y el largo plazo, y la compañia opera sin grandes sobresaltos bajo relaciones más bien formales. Es una cultura que apuesta y confia en la empresa (mineria, energía, seguros, bancos).

Finalmente, en ambientes de bajísimo riesgo por su protección, y de lenta retroalimentación de resultados, donde la gente tiene dificultades para medir lo que hace y su entorno está regulado, se genera una cultura de poca actividad, concentrada en el cómo, en los procedimientos y en el dominio de los procesos administrativos. Es una cultura centrada en el proceso, burocrática (servicios y empresas publicas/gubernamentales, industriales monopólicas/reguladas).

Como vemos, son variadas las situaciones en las cuales se requiere usar el concepto de cultura organizacional, sin embargo, la apertura y la globalización del mercado traen nuevos competidores, lo cual implica contar con empresarios audaces, flexibles y sin culturas fuertes que los aten; se deben sacudir de normas y conductas tradicionales para lograr consolidar un equipo humano con excelente capacidad de respuesta, para ejecutar los procesos de activación de los servicios.

\section{EVALUACIÓN DE LA CALIDAD DE SERVICIO}

Hacer efectiva la calidad significa que las personas de las distintas divisiones y departamentos han de cooperar para comenzar a desarrollar un programa continuo de calidad, deben estar en estrecha comunicación unos con otros y deben hacer su trabajo con una alta calidad de rendimiento; tienen que trabajar juntos para resolver los problemas y prever las dificultades.

Esto implica un estudio intensivo de todas las operaciones de servicio, controlarlas continuamente para descubrir los problemas y medir el progreso.

La propuesta que se presenta en el siguiente sistema de evaluación nos permitirá medir la calidad de servicio en una organización: 


\section{Objetivo}

\section{Diagnóstico}

\section{Nivel externo}

Nivel interno

- mercado

- autoevaluación

- cliente

- prestación del servicio

Cruce de información

\section{Evaluación del nivel de calidad}

Identificación del problema

\section{Planteamiento de estrategias}

\section{Objetivo}

Delimitar qué es lo que se desea investigar, en este caso la calidad de atención en una organización de servicios.

\section{Diagnóstico}

El diagnóstico se realiza en dos niveles: externo e interno:

\subsection{Nivel externo}

a) Investigar el nivel de percepción del cliente con respecto a la organización.

Cómo percibe el servicio:

- cumplimiento de lo prometido por la organización,

- satisfacción del cliente,

- de acuerdo con los canales de retroalimentación.

Qué imagen tiene de la organización.

b) Investigar cuáles son las expectativas del cliente con respecto al servicio.

c) Obtener datos sociodemográficos como: edad, sexo, educación, residencia.

d) Conocer gustos, preferencias y necesidades del cliente. 
e) Cuál es la ubicación de nuestra organización con respecto a la competencia.

\subsection{Nivel interno}

a) Se debe conocer el objetivo de la organización, la labor que realiza y la infraestructura.

b) Tecnología que se utiliza en el servicio al cliente.

c) Posición de la gerencia frente a las necesidades del cliente.

d) Personal de la organización.

- Situación del personal.

- Reclutamiento.

- Capacitación.

e) Políticas de la empresa en:

- El establecimiento de normas y servicios.

- La especificación de prestación de servicios.

f) Qué tipo de relaciones se establecen entre normas y prestación de servicios.

g) Tipo de diseño de servicio al cliente.

Los instrumentos que se utilizarán para la recolección de datos y que permitirán realizar la evaluación de la calidad son:

- A nivel cuantitativo: usar datos estadísticos sobre indice de ventas, afluencia de público a horas punta, encuestas para conocer el posicionamiento de imagen de la organización, ubicación de preferencia del público, gustos, preferencias y expectativas.

- A nivel cualitativo: las entrevistas sobre el servicio brindado, la observación participante y los focus group.

- Datos cualitativos que pasan a ser cuantitativos, sistema de reclamos y quejas, buzón de sugerencias, llamadas por teléfono sobre el servicio.

Es necesario realizar mediciones con diferentes herramientas para obtener la mayor objetividad.

\section{Cruce de información}

El diagnóstico de calidad a nivel interno y externo nos debe dar información del nivel de calidad de servicio y de las fallas que se están suscitando al momento de su prestación. 


\section{Identificación del problema}

Con esa información se puede identificar el problema y ubicar en qué parte de la organización se está produciendo.

Es decir, retroalimentamos el diagnóstico tanto a nivel interno como a nivel externo y ubicamos el problema.

Muchas veces la información que obtenemos nos muestra problemas en:

- La coordinación con los diferentes niveles de la organización.

- No se conoce al cliente y se diseña un servicio que no va de acuerdo con sus necesidades.

- No hay compromiso para llevar las medidas de corrección adecuada.

- No se establecieron patrones adecuados para desempeñar la función.

- Falta de capacitación para atender al cliente.

- Falta de tecnología.

- La empresa promete y no cumple (la expectativa crece y lo que se percibe es menor).

\section{Planteamiento de estrategias}

Una vez ubicado el problema se pueden plantear estrategias que permitirán alcanzar el o los objetivos trazados, por ejemplo:

- Definir con claridad las normas de rendimiento.

- Siempre que sea posible, exponer los resultados de la evaluación donde todos puedan verlos.

- Suministrar comentarios y reacciones rápidas y frecuentes sobre el rendimiento.

- Utilizar tecnología adecuada para los clientes (la tecnología sólo es valiosa si resuelve sus problemas).

- Invertir en investigación de los clientes.

- Mantenerse en contacto con los clientes (ayudará a reconocer las oportunidades de servir y a desarrollar una relación más estrecha con el público).

- No suponer cuáles son las necesidades del público sino preocuparse de descubrirlas (ver el servicio desde la perspectiva del cliente).

- Encontrar y contratar gente que se preocupe por dar servicio de calidad.

- Concentrar los esfuerzos en la formación y la motivación del personal de servicio de manera que sepan cómo y por qué prestan sus servicios. 
En conclusión, el servicio vive y muere con las transacciones individuales que realice cada empleado en el contacto directo con el público, pero además, para que exista el servicio de calidad, la alta dirección de la empresa deberá participar directamente en él, demostrando su compromiso por medio de hechos, de la participación personal en los programas de servicio y manteniendo un estrecho contacto con los empleados responsables de poner en marcha el servicio de calidad. 
Bibliografía

Gabett, Thomas

1991

Kullmer, Jorge

1993

Luft, Joseph

1969

Rossander, A.C.

1992

Tello, Max et al.

1994

Terry [y] Franklin

1986

Tolela Myers

1983

Universidad de Lima 1994
Imagen Corporativa. Colombia: Editorial Legis.

Gerencia No 201, marzo-abril, p. 29

La interacción humana. National Press Book

La búsqueda de la calidad en los servicios. Ediciones Díaz Santos.

Empresa y comunicación. El estado de la comunicación empresarial en el Perú. Lima: Universidad de Lima. Serie Cuadernos Cicosul $N^{\circ} 17$.

Principios de Administración. México: Compañía Editorial Continental.

Administración mediante la comunicación. Un enfoque organizacional. México: McGraw Hill.

Memoria del IV Congreso Internacional de Desarrollo Organizacional y I Congreso Nacional de Calidad Total. Lima: Universidad de Lima, Facultad de Ciencias Administrativas. 